\title{
CAMBIOS ESPACIO-TEMPORALES COSTEROS CON IMÁGENES LANDSAT 8 Y SENTINEL 2 (2015-2019) EN CHILE CENTRAL; PLAYA REÑACA, BAHÍA DE CONCÓN Y BAHÍA DE ALGARROBO
}

\author{
COASTAL SPATIAL-TEMPORAL CHANGES WITH LANDSAT 8 AND SENTINEL2 IMAGINE (2015-2019) IN \\ CENTRAL CHILE; REÑACA BEACH, CONCÓN BAY AND ALGARROBO BAY
}

Idania Briceño de Urbaneja ${ }^{a, b,{ }^{*}}$, Elena Sánchez-García ${ }^{c}$, Josep E. Pardo-Pascual ${ }^{b}$, Jesús PalomarVázquez ${ }^{b}$, Raúl Ugalde-Peraltaa ${ }^{a}$ Catalina Aguirre-Galaz ${ }^{d}$, Waldo Pérez-Martínez ${ }^{\mathrm{a}, \mathrm{b}}$, Paulina VidalPáez $z^{a, b}$

\author{
a Centro de Observación de la Tierra (Hémera), Universidad Mayor, Camino La Piramide 5750, 8580745 Santiago, Chile. \\ Idania.briceno@umayor.cl; raul.ugalde@umayor.cl; waldo.perez@umayor.cl; paulina.vidal@umayor.cl \\ ${ }^{b}$ Geo-Environmental Cartography and Remote Sensing Group (CGAT), Universitat Politècnica de València, Camino de Vera s/n, 46022 \\ Valencia, España. jepardo@cgf.upv.es; jpalomav@upvnet.upv.es \\ ${ }^{c}$ Research Institute of Water and Environmental Engineering (IIAMA), Universitat Politècnica de València, Camino de Vera s/n, 46022 \\ Valencia, España. elsncgar@upv.es \\ d Escuela de Ingeniería Civil y Oceánica, Universidad de Valparaíso, Chile. catalina.aguirre@uv.cl
}

\begin{abstract}
:
Due to their oceanographic and climatic ecological characteristics, the coastal areas of the Valparaíso Region of Chile are of great importance for the country's tourist, economic, and environmental development. The region's coasts have been affected by extreme events since 2015 , with recurrent swells that have increased between $10 \%$ and $25 \%$ compared to previous years, causing the interruption of the annual dynamic process of the beaches accentuated by anthropic interventions on beaches, wetlands and coastal dunes that promote coastal degradation. This work aims to characterize which part of the beaches and at what time of year there is a higher risk of severe erosion in the immediate areas to the coast and explain its causes based on satellite images of medium resolution satellite images. 128 SDS were extracted with Landsat $8 \mathrm{OLI}$ and Sentinel 2 images with systems SHOREX. Average widths calculated for the series in each of the seasons. Recording the minimum widths in the proximal areas with averages of $12.3 \mathrm{~m} ; 23.6 \mathrm{~m}$ and $21.2 \mathrm{~m}$ Concón Bay, Reñaca Beach and Algarrobo bay, respectively showing critical or problematic states. The dominant direction of the waves and the geographical disposition of the three beaches seems evident that there is a robust longitudinal transport to the north that generates less sediment accumulation in the proximal areas. The seasonal variation of the beaches distinguishes by more energetic conditions in winter than in summer. But they are also influenced by rapid episodic periods, low atmospheric pressure systems from the north and south, swells and tropical storms. Hence, it is unlikely that the variations in the width of the beach are only due to winter-summer energetic conditions.
\end{abstract}

Key words: coastal dynamics, coastline, erosion, critical areas, multitemporal coastal mapping, remote sensing

\begin{abstract}
Resumen:
Las áreas costeras de la región de Valparaíso de Chile por sus características oceanográficas, climáticas y ecológicas son de gran importancia para el desarrollo turístico, económico y ambiental del país. Las costas de la región se han visto afectadas por eventos extremos desde 2015, con marejadas recurrentes que han aumentado respecto a años anteriores entre el $10 \%$ y $25 \%$. Esto ha provocado la interrupción del proceso dinámico anual de las playas, acentuado por las intervenciones antrópicas sobre estos ambientes costeros, humedales y dunas que promueven su degradación. El trabajo que se presenta tiene como objetivo caracterizar en qué parte de las playas y en qué momento del año hay un riesgo más alto de erosión grave en las áreas inmediatas a la costa, así como tratar de explicar sus causas apoyándose en la utilización de imágenes satelitales de resolución media. Se extrajeron 128 SDS con imágenes Landsat 8 OLI y Sentinel 2 con el sistema SHOREX. Se calcularon los valores promedio de anchura de playa para la serie en cada una de las temporadas registrando anchuras mínimas en las zonas proximales de 12,3 m; 23,6 m y 21,2 m para la Bahía de Concón, Playa Reñaca y Bahía de Algarrobo respectivamente, demostrando estados críticos o problemáticos. La dirección dominante del oleaje y la disposición geográfica de las tres playas hace evidente la existencia de un fuerte transporte longitudinal hacia el norte que genera que las áreas proximales presenten menor acumulación de sedimentos. La variación estacional de las playas se caracteriza por condiciones más energéticas en invierno que en verano. No obstante, se ven también influenciadas por periodos episódicos rápidos, sistemas de bajas presiones atmosféricas del norte y del sur, así como marejadas y tormentas tropicales que se suman al moldeamiento de las playas.
\end{abstract}

Palabras clave: dinámica costera, línea litoral, erosión, áreas críticas, cartografía multitemporal de costas, teledetección

`Corresponding Author: Idania Briceño de Urbaneja, idania.briceno@umayor.cl 


\section{Introducción}

Las costas de la Región de Valparaíso por su ubicación son muy atractivas para diferentes actividades económicas, emplazamiento de población y desarrollo regional. Desde hace 30 años las acciones antrópicas han estado provocando el deterioro de los ambientes marino-costeros (Martínez et al. 2019), mediante la construcción de infraestructuras sobre playas, humedales y dunas (Rangel-Buitrago et al. 2018; Martínez et al. 2018).

Las costas son ambientes que constantemente están cambiando, debido a las interacciones entre el océano, la atmósfera, la tierra y las personas (Nicholls 1995).

La monitorización del cambio de la posición de la línea de costa es importante para el desarrollo de actividades económicas, planificación regional, recreación y protección ambiental (Sekovski et al. 2014). Es un indicador que permite cuantificar, zonificar áreas con peligros naturales, revelar procesos de erosión-acreción, detectar áreas críticas, estudiar los patrones sedimentarios, componentes bióticos y abióticos presentes, entender la morfodinámica costera, entre otros (Gens 2010).

A lo largo de la región de Valparaíso, el litoral arenoso desde 2015 ha sido frecuentemente afectado por diversos eventos extremos, tales como: tormentas, marejadas e inundaciones. En los últimos años se ha manifestado el aumento de marejadas en temporadas de primavera-verano respecto a años anteriores, con un incremento que oscila entre $10 \%$ y $25 \%$ según los pronósticos (https://marejadas.uv.cl/), convirtiéndose en la principal amenaza para la región (Igualt et al. 2019). Adicionalmente, desde 2010 la región central de Chile atraviesa por una sequía extrema, con registros de disminución de precipitación entre $25 \%$ a $45 \%$ en las secciones altas de las cuencas que aportan sedimentos a la costa. Esto ha generado la reducción de la carga sedimentaria de los ríos hacia las costas en hasta un $90 \%$ (Garreaud et al. 2020; Masotti et al. 2018).

Ante esta problemática, surge la necesidad de estudiar las costas con metodologías y herramientas robustas y estables que permitan monitorizar en periodos cortos los cambios descritos, pues la región de Valparaíso se enfrenta a diversas amenazas asociadas a cambios rápidos en la geomorfología costera.

Las técnicas tradicionales para establecer la posición de la línea de costa requieren, una amplia inversión en tiempo y recursos y es costoso abarcar grandes superficies costeras (Sunder et al. 2017). En Chile se han realizado estudios espacio-temporales de la variabilidad interanual e interdecenal de la posición de la línea de costa, utilizando técnicas fotogramétricas a múltiples escalas, con modelos de ajustes espiral logarítmica, sistemas de información geográfica, levantamientos topográficos y modelamiento a través de los forzamientos oceánicos con énfasis en eventos puntuales de marejadas, temporales y tsunamis (Martínez 2011; Martínez et al. 2016, 2018; Winckler et al. 2017). Con estas técnicas se ha concluido que el $80 \%$ del litoral arenoso en Chile presenta problemas de erosión, especialmente las playas con campos dunares y humedales (Ministerio del Ambiente 2019).
En las últimas décadas las imágenes multiespectrales, se han convertido en una fuente robusta y estable de datos para el seguimiento sistemático de las costas, debido a su gran cobertura, resolución espacial y temporal, así como su libre disponibilidad en los portales del Servicio geológico de los Estados Unidos (USGS) y la Agencia espacial europea (ESA).

Este acervo de información espacial ha estimulado el desarrollo y aplicación de métodos automáticos y semiautomáticos para la extracción de líneas de costa y la aplicación de múltiples metodologías y escalas: TasseledCap (Scott et al. 2003), redes neuronales (Mas 2004), índices espectrales (Feyisa et al. 2014; Liu et al. 2017). Con frecuencia dichas metodologías han requerido utilizar vehículos aéreos no tripulados con resolución espacial centimétrica (Zanutta et al. 2020), LiDAR aerotransportado (Kim et al. 2017) y, comúnmente, con satélites de resoluciones medias de la constelación Landsat MSS, TM, ETM, OLI y Sentinel 2 (Cabezas-Rabadán et al. 2017; Cabezas-Rabadán and Pardo-Pascual 2017; Sánchez-García et al. 2019, 2020; Vos et al. 2019).

Este trabajo tiene como objetivo caracterizar en qué parte de las playas y en qué momento del año hay un riesgo más alto de erosión grave en las áreas inmediatas a la costa, así como tratar de explicar sus causas, apoyándose en la utilización de imágenes satelitales de resolución media.

\section{2. Área de estudio}

La Bahía de Concón, la playa de Reñaca y la Bahía de Algarrobo se ubican en la Región de Valparaíso, Chile Central (Fig. 1). Son lugares de interés para comprender la influencia del océano, agentes atmosféricos y antrópicos en la dinámica costera. Tienen una longitud de 3,$3 ; 1,3$ y $4,5 \mathrm{~km}$ respectivamente. Las playas están orientadas aproximadamente de norte a sur y suelen estar expuestas a marejadas persistentes de alta energía del Océano Pacífico (Agredano et al. 2019). Concón incluye un extenso campo de dunas costaneras, principalmente de los tipos barjan y longitudinal, con edades pleistocenas tardías y holocenas. Reñaca tiene una morfología cambiante, mostrándose más plana como consecuencia de las marejadas. Algarrobo se caracteriza por un extenso litoral arenoso en sus zonas central y distal, con aportes sedimentarios locales a través de los estuarios El Membrillo y San Jerónimo (Martínez 2007).

\section{Metodología}

Se escogieron y contrastaron 3 playas con características distintivas entre sí: playas con dunas y desembocadura de río, playa urbana, playa con construcciones sobre humedales costeros.

\subsection{Extracción automática de posición de la línea de costa}

El sistema de extracción de la posición de líneas de costas propuesto (Pardo-Pascual et al. 2012, 2018; Almonacid-Caballer et al. 2016; Palomar-Vázquez et al. 2018, Sánchez-García et al. 2019) sistematiza en tres bloques de trabajo a realizar mediante la descarga, preprocesamiento y procesamiento de grandes volúmenes de información espacial georreferenciada, y 
extracción a nivel subpíxel de la posición de la línea de costa a partir de imágenes de resolución media (Landsat $5,7$ y 8 y Sentinel 2$)$.

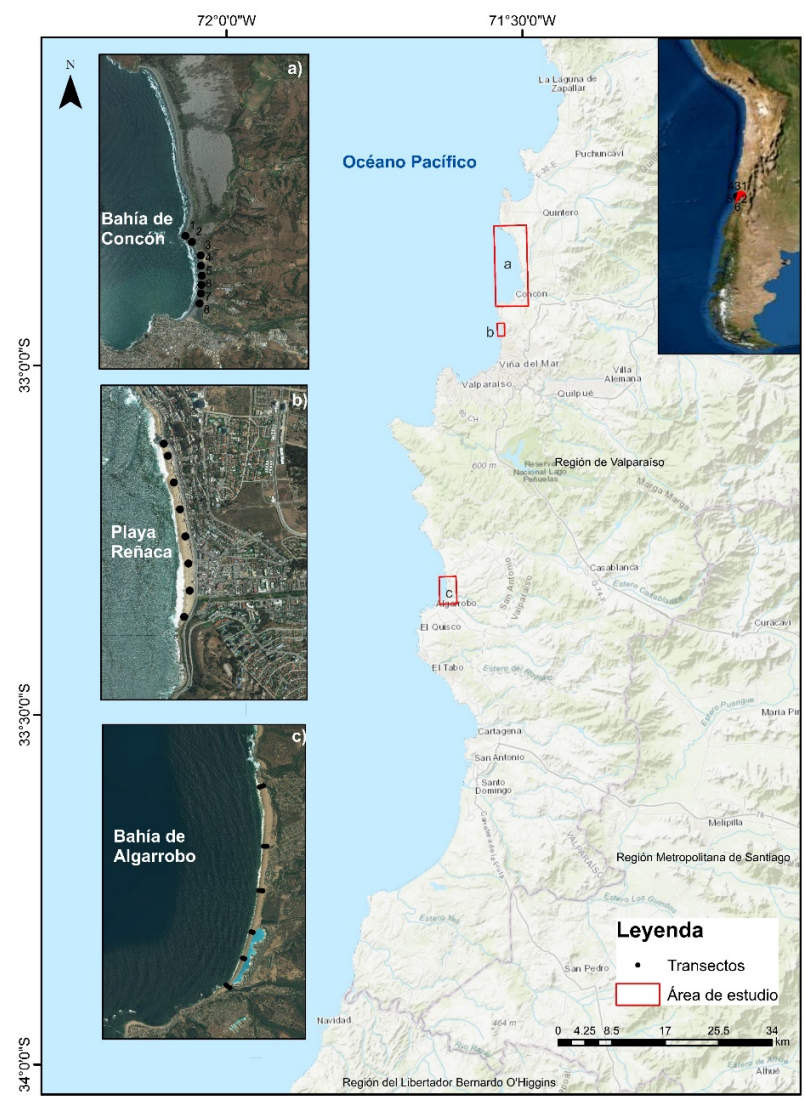

Figura 1: Ubicación de las tres zonas de estudio. Los círculos negros corresponden a transectos perpendiculares. De norte a sur: a) Bahía de Concón; b) Playa Reñaca y c) Bahía de Algarrobo.

En este trabajo, se utilizó el sistema SHOREX de Palomar-Vázquez et al. (2018). Se emplearon las bandas SWIR1 1,57-1,65 $\mu \mathrm{m}$ y 1,56-1,65 $\mu \mathrm{m}$ de Landsat 8 OLI y Sentinel 2 respectivamente. En la (Fig. 2) se muestra la distribución temporal de las 128 SDS utilizadas en este estudio.

\subsection{Ancho de la playa}

Se estableció una línea interior fija para determinar el ancho de la playa. Esta línea se digitalizó a partir de imágenes de alta resolución espacial $(0,5 \mathrm{~m})$ usándose como criterio de definición el borde de las dunas, infraestructura de caminerías y calzadas (Fig. 3).

Los anchos de la playa se analizaron en segmentos de $100 \mathrm{~m}$ (Fig. 4). Se calcularon los promedios por temporadas de los anchos de la línea de costa para evidenciar la variabilidad temporal de las SDS.

La categorización de los anchos de la playa se estableció según los criterios (Cabezas-Rabadán et al. 2019) como sigue: Playas < $15 \mathrm{~m}$ críticas, entre 15-30 m problemáticas, y $>30 \mathrm{~m}$ no problemáticas.

\subsection{Oleaje}

En Chile no existen registros de oleaje de largo plazo, por lo que se han llevado a cabo simulaciones numéricas utilizando el modelo espectral Wavewatch III desarrollado por el NOAA/NCEP, de la Universidad Técnica de Delft y NASA.

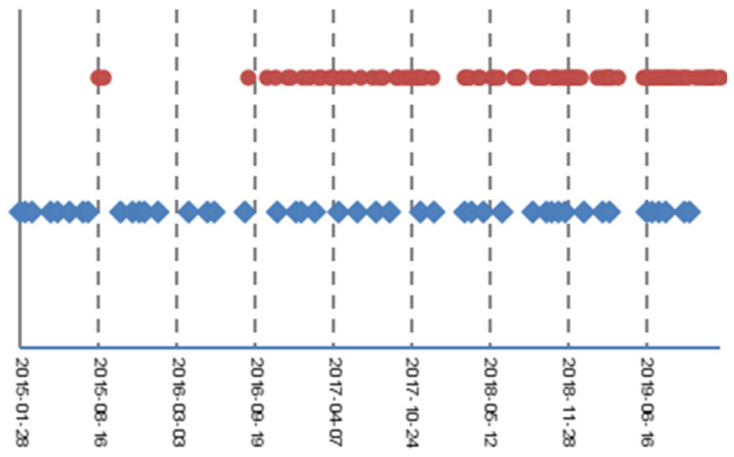

Figura 2: Distribución temporal de SDS obtenidas de las imágenes Landsat 8 (rombos azules) y Sentinel 2 (puntos rojos).
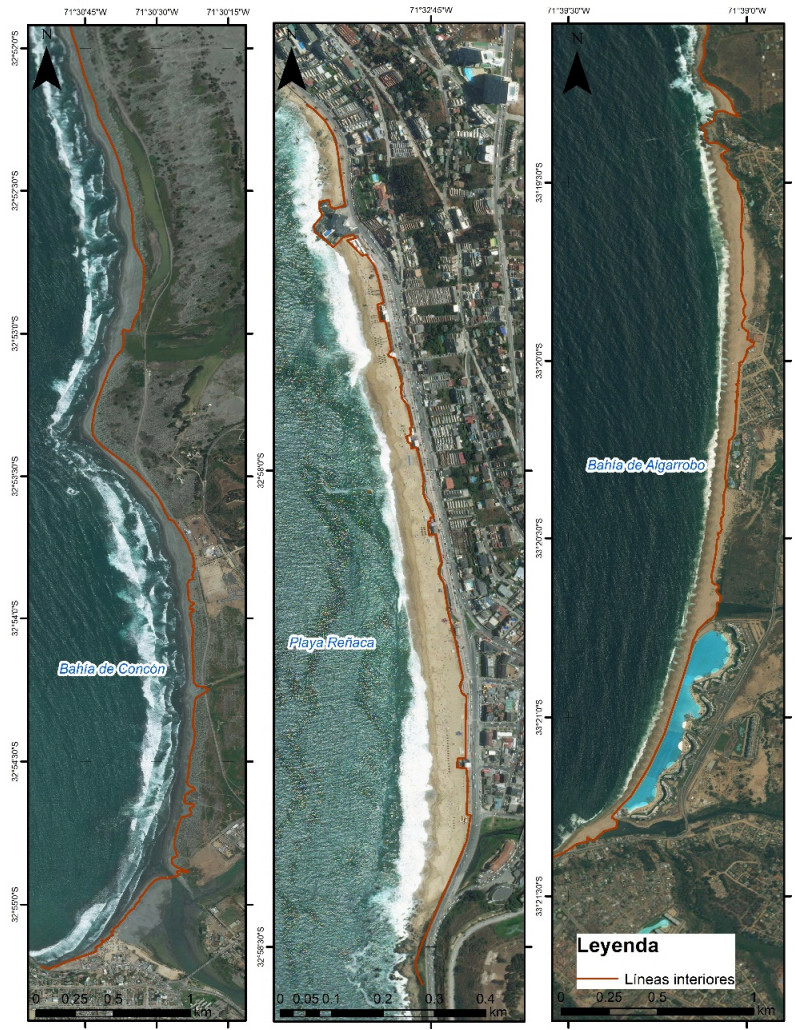

Figura 3: Delimitación línea interior de la playa

Para realizar simulaciones del oleaje que incide en las costas de Chile es necesario contar con un dominio espacial que abarque toda la cuenca del océano Pacífico (Fig. 5a). En esta configuración, el dominio tiene una resolución espacial de un grado. Para la creación de la batimetría, máscaras de obstrucción y grilla computacional se utilizó el algoritmo de generación de mallas GridGen (Chawla et al. 2013). La base de datos batimétrica utilizada para generar condiciones de borde de fondo es ETOPO2, la cual cuenta con una resolución espacial horizontal de $\sim 4 \mathrm{~km}$ (Fig. 5b). Para generar condiciones de borde laterales, se utilizó la línea de costa de la base de datos GSHHS (A Global Self-consistent, Hierarchical, High-resolution Shoreline Database). 


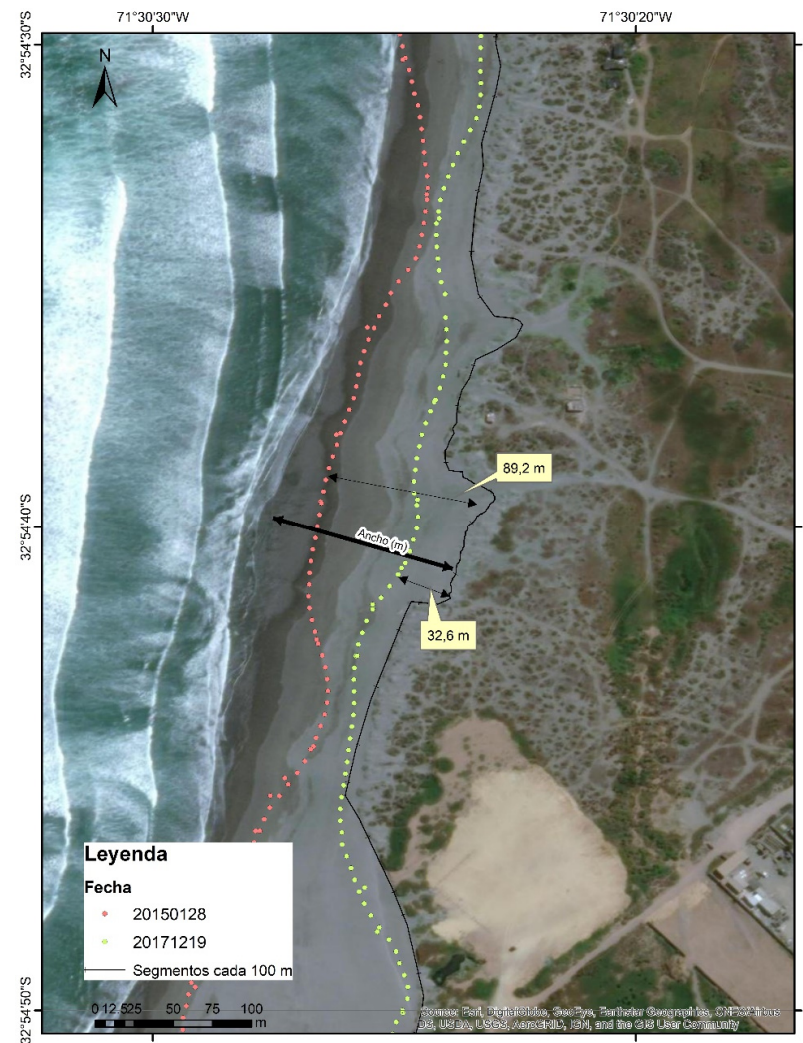

Figura 4: Diagrama de medición de ancho de la playa.

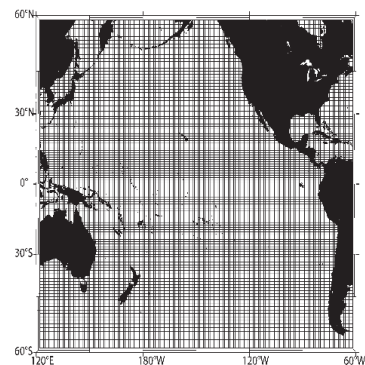

(a)

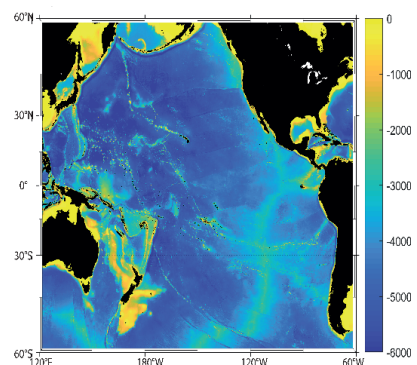

(b)
Figura 5: Modelación de oleaje: a) Malla de cálculo; b) batimetría (ETOPO2) utilizada.

Como condición de borde superficial, el modelo fue forzado por el viento del reanálisis ERA5 (Fig. 6), perteneciente al Centro Europeo de Pronóstico Meteorológico (ECMWF, por sus siglas en inglés). Este reanálisis posee aumentos en la resolución de las capas verticales, aumentos en la resolución horizontal de $80 \mathrm{~km}$ a $31 \mathrm{~km}$, y adicionalmente asimila más conjuntos de
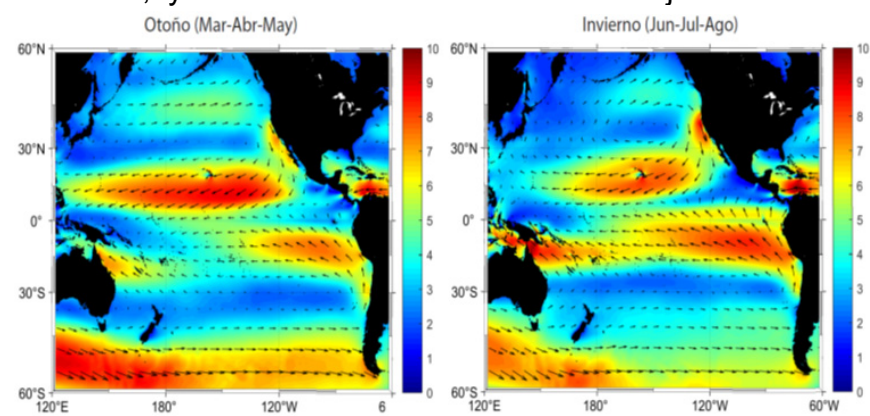

datos de observación que los reanálisis anteriores (Hersbach et al. 2020). Datos de la velocidad zonal y meridional del viento a $10 \mathrm{~m}$ sobre la superficie con una resolución temporal de $6 \mathrm{~h}$ fueron utilizados. Además, se incorpora la concentración de hielo marino del reanálisis ERA5, considerando un umbral de $30 \%$ de hielo para distinguir el punto de grilla como tierra. Si la concentración de hielo cae bajo el umbral, el punto de grilla es reactivado (Chawla et al. 2013).

\section{Resultados y Discusión}

\subsection{Extracción automática de la línea de costa}

Se obtuvo 128 posiciones de la línea de costa entre los años 2015-2019, 46 con imágenes Landsat 8 OLI de 30 $\mathrm{m}$ de resolución y, 82 con imágenes Sentinel 2 de $20 \mathrm{~m}$. Uno de los problemas de la utilización de imágenes ópticas en Chile está relacionado con la vaguada costera, que genera un complejo patrón de nubosidad sobre las costas. Por esta razón, se descartaron las SDS donde se produce confusión de la orilla con las sombras de edificios, acantilados rocosos y sombras de nubes. Esto se resolvió parcialmente aplicando para las imágenes Sentinel 2 el producto Scene Classification (SC) y las bandas en nivel Bottom of the Atmosphere (BOA). Para las imágenes Landsat se resolvió a través del índice espectral AWEINSH propuesto por (Feyisa et al. 2014).

El sistema SHOREX ha sido utilizado para la extracción de líneas de costas en entornos micromareales de baja energía en playas del Mediterráneo y entornos mesomareales del Atlántico (Cabezas-Rabadán et al. 2017, 2019). La información que se extrae con este sistema es la más estable en cuanto a resolución espacial, periodos de revisita y análisis de series temporales. Permitiendo la comparabilidad de los datos en series largas y con errores de precisión subpíxel.

\subsection{Anchos de las playas}

El ancho de la playa a menudo exhibe una variación intraanual que se ve afectada por sistemas de bajas presiones en latitudes medias de los hemisferios sur y norte, marejadas durante el invierno, anticiclón y tormentas tropicales (Beyá et al. 2016). Los procesos naturales como las mareas, la fijación de bancos, la migración de canales, así como la influencia antropogénica, pueden afectar directamente la morfología y, en consecuencia, el ancho de la playa (Wijnberg 2002).
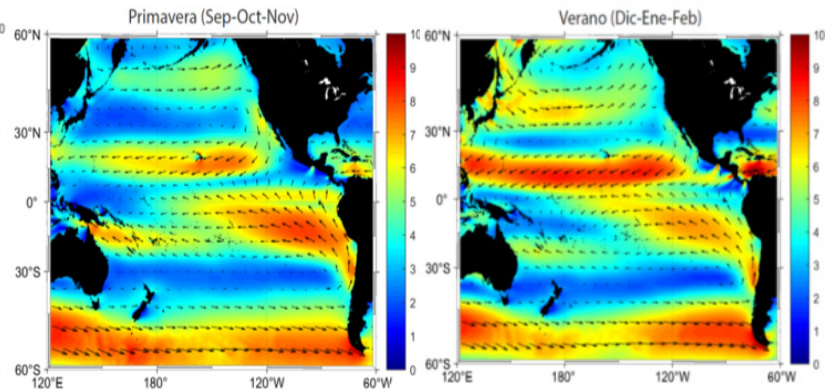

Figura 6: Climatología del viento a $10 \mathrm{~m}$ de altura sobre el Océano Pacífico del reanálisis ERA5 (ms $\left.{ }^{-1}\right)$. La velocidad del viento es mostrada en colores y su dirección en vectores. Datos cada $6 \mathrm{~h}$ han sido utilizados para forzar el modelo de oleaje Wavewatch III. 
En el área de estudio los anchos críticos con valores inferiores a $15 \mathrm{~m}$ se localizan en la zona proximal. En la Bahía de Concón con un promedio de $12,3 \mathrm{~m}$ y una desviación estándar de 10,5 m. En la desembocadura del río Aconcagua las variaciones de los anchos oscilan en promedio $73,6 \mathrm{~m}$, desviación estándar de $32,9 \mathrm{~m}$ y ancho máximo de $157 \mathrm{~m}$, este sector muestra fuerte dinámica sedimentaria (Martínez 2007). Las zonas central y distal de la bahía de Concón se mantuvieron estables.

En Reñaca por su ubicación y exposición muestra valores de anchos de playa variables, en algunos casos determinados por marejadas en agosto de 2015 que movilizaron grandes volúmenes de sedimentos en la zona distal de la playa provocando grandes cambios morfológicos (Agredano et al. 2019). Los eventos extremos de oleaje (denominados comúnmente marejadas) que se propagan desde el $\mathrm{W}$ y NW tienen el potencial de producir grandes impactos a lo largo del borde costero, particularmente en sectores de las bahías abiertas al NW (Campos-Caba 2016).

El valor promedio del ancho de playa en la zona proximal es de 23,6 m, el valor máximo 54,2 m, y una desviación estándar de 9,24 m. En la zona distal de la playa los valores promedio son $38,6 \mathrm{~m}$, máximo $70,2 \mathrm{~m}$ y desviación estándar de $12 \mathrm{~m}$.

La bahía de Algarrobo en su zona proximal presenta valores promedios de 21,2 $\mathrm{m}$, desviación de 9,95 $\mathrm{m}$ y valor máximo de $57 \mathrm{~m}$. Las zonas centrales y distales se mantuvieron estables.

Dada la dirección dominante de los oleajes y la disposición geográfica de las tres playas parece evidente que existe un fuerte transporte longitudinal hacia el norte que genera que las partes proximales (meridionales) de las mismas sean las que puedan sufrir un déficit de sedimentos principalmente si disminuyen los aportes externos. Ello explicaría la menor anchura de las tres playas en dichos sectores (Fig 7).

Los ciclos estacionales de la playa generalmente se atribuyen a variaciones en el nivel de energía de las olas incidentes, lo que resulta en playas anchas y sin barreras en verano, junto a playas estrechas y con barras en invierno (Masselink and Pattiaratchi 2001). Las costas a lo largo de Valparaíso son muy variables tanto espacial como temporalmente. Atraviesan períodos de erosión episódica rápida, seguida de años intermedios, a décadas durante las cuales se reconstruyen las playas y las dunas. Los procesos naturales de recuperación de playas son los que se han visto alterados en los últimos años, debido al aumento de las marejadas en temporadas de primavera y verano.

\subsection{Oleaje}

Las series de tiempo de los parámetros del oleaje muestran una importante variabilidad intraestacional e interanual. Esta última ha sido relacionada principalmente a la variabilidad climática que introduce $\mathrm{El}$ Niño, Oscilación del Sur y el Modo Anular Austral (e.g. Hemer et al. 2010; Stopa and Cheung 2014; Aguirre et al. 2020).

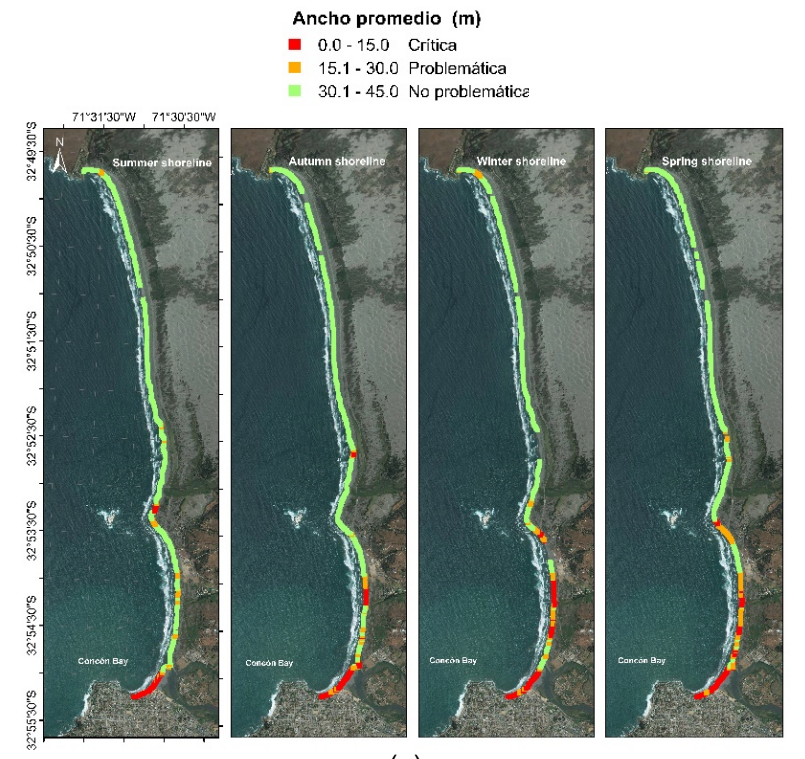

(a)
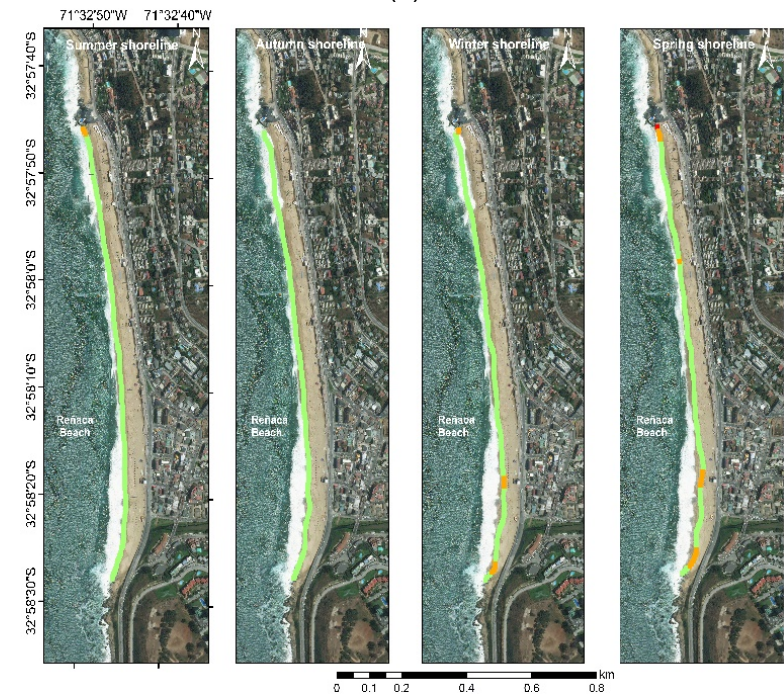

(b)
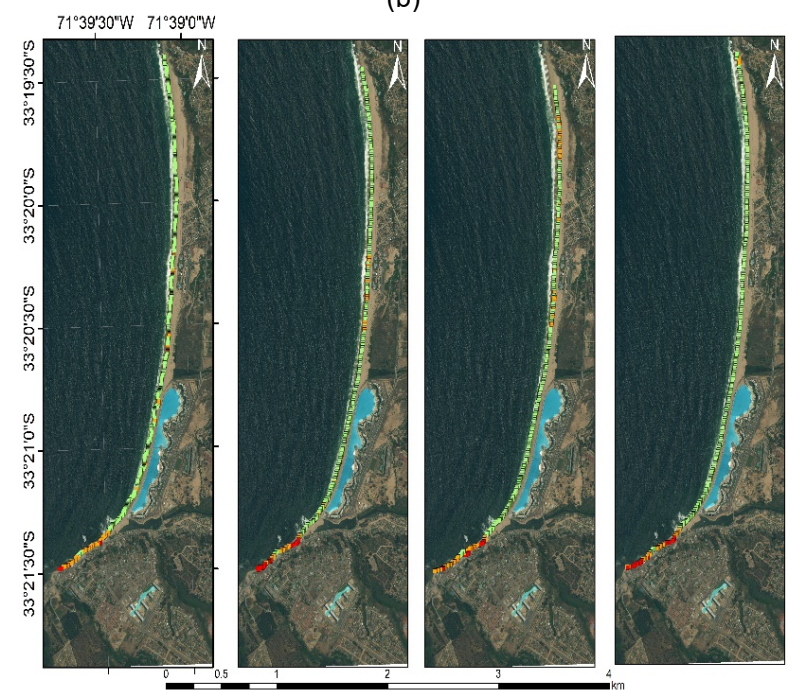

(c)

Figura 7: Variabilidad temporal del ancho de la línea de costa de izquierda a derecha verano, otoño, invierno y primavera: a) Bahía de Concón; b) Playa Reñaca; c) Bahía de Algarrobo. Color Rojo Playas críticas anchos < 15 m. Color Naranja Playas problemáticas anchos $15-30 \mathrm{~m}$ y color verde playas con anchos superiores a $30 \mathrm{~m}$. 
La región de Valparaíso, en particular, muestra una baja variabilidad estacional en los parámetros promedio de altura del oleaje (Fig 8). La altura significativa se encuentra en torno a los $2,5 \mathrm{~m}$ y presenta un cambio estacional promedio del orden de $10 \mathrm{~cm}$ (Fig. 8a). Algo similar ocurre con los períodos, los cuales se encuentran en el orden de 10 segundos, y tienen una variación estacional promedio del orden de 1 segundo (Fig. 8b) siendo ligeramente mayor en los meses invernales y primaverales. La climatología de la dirección del oleaje muestra la dominancia de las olas propagándose desde el SW $\left(225^{\circ}\right)$, la cual presenta una variación promedio de $10^{\circ}$ (Fig. 8c).

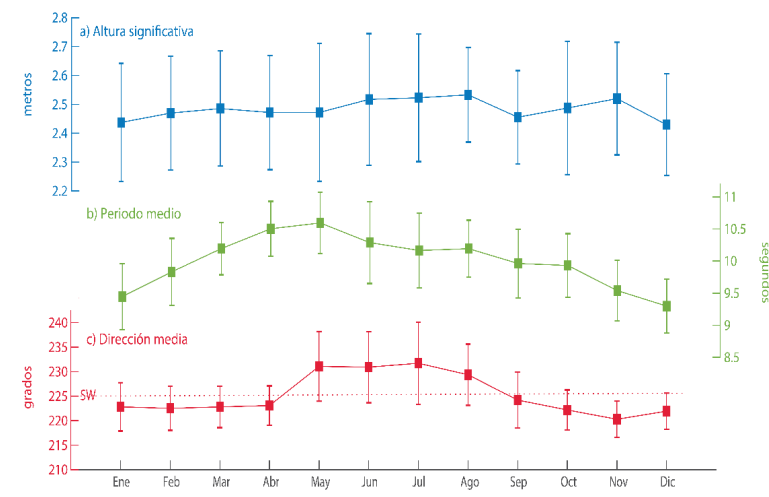

Figura 8: Climatología de parámetros del oleaje frente a la costa de Valparaíso $\left(33^{\circ} \mathrm{S} ; 73^{\circ} \mathrm{W}\right)$ : a) altura significative; b) periodo medio; c) dirección media, la línea segmentada indica el SW. Los datos de oleaje han sido aportados por el IHC de

Cantabría y se basan en la modelización por reanalisis de Pérez et al. (2017).

Los parámetros de resumen del oleaje pueden esconder cambios que se producen en el estado del mar, ya que, por ejemplo, distintos espectros de energía de oleaje pueden conducir a un mismo resultado de altura significativa.

La energía dominante desde el SW presenta una disminución durante el verano, consistente con la disminución de la intensidad de los vientos sobre el Océano Austral y el desplazamiento de las trayectorias de tormentas hacia mayores latitudes. Sin embargo, durante el verano podemos ver un máximo de energía que tiene una dirección de propagación desde el NW y centrado en una frecuencia de $0.07 \mathrm{~Hz}$. Este máximo de energía en el espectro está asociado al aumento de la intensidad de los ciclones extratropicales en el hemisferio norte (Fig 9).

Si bien los patrones climatológicos calculados muestran un dominio del oleaje en el modelado costero, los eventos puntuales también han sido ejes caracterizadores de los cambios más abruptos en las playas de estudio. Un caso que refleja este escenario es la marejada acontecida en la Bahía de Algarrobo el 15 octubre de 2020, donde las construcciones sobre humedales y dunas costaneras sufrieron importantes daños (Fig 10). Esto avala la condición de susceptibilidad de las estructuras en esta y otras playas del litoral central chileno.

\section{Conclusiones}

El sistema SHOREX permite realizar estudios multitemporales robustos y estables de las playas a diferentes escalas espacio-temporales. La nubosidad es sin duda una limitación en la adquisición de imágenes ópticas. Sin embargo, en comparación con otras técnicas fotogramétricas, el trabajo con estas imágenes de satélite de resolución media sigue siendo el procedimiento más eficiente para la monitorización de los cambios en las costas y ambientes marino-costeros.
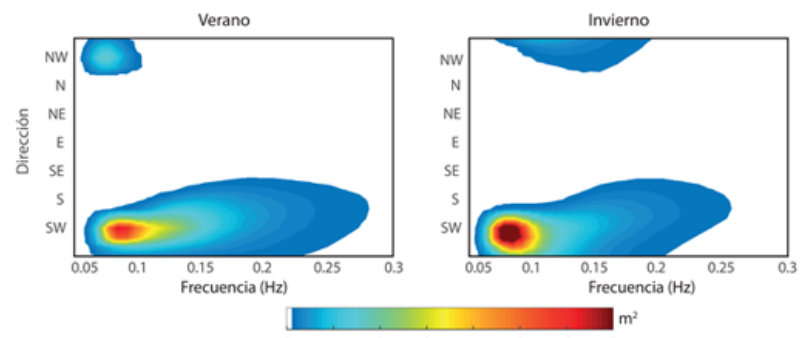

(a)

(b)

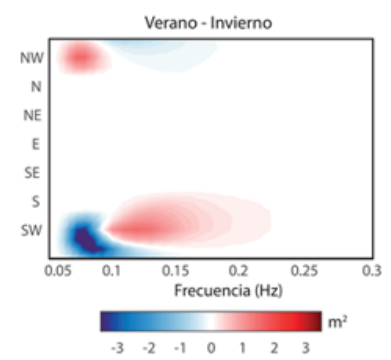

(c)

Figura 9: Espectros de energía del oleaje: a) Climatología de verano; climatología de invierno; c) diferencia entre verano e invierno.

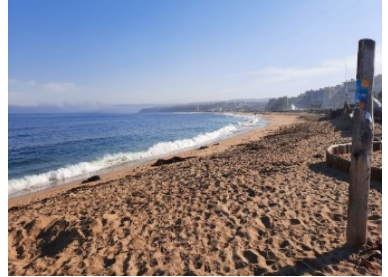

(a)

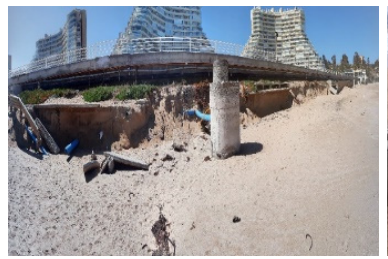

(c)

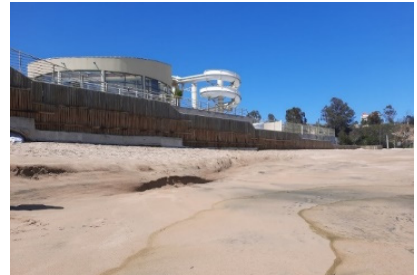

(b)

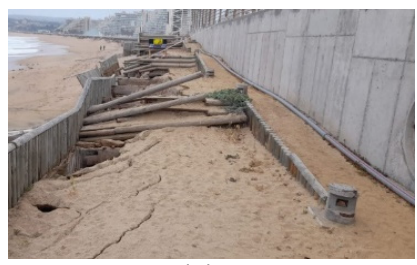

(d)
Figura 10: Playa de San Alfonso del Mar (Algarrobo) antes y post evento de marejada: a) 05/03/20200; b) 08/10/2020; c) 08/10/2020; d) 30/10/2020

Los anchos medios por temporadas representan la situación general de las playas en el conjunto de datos analizados, sin embargo, hay eventos puntuales $y$ extremos que no se ven reflejados en estos valores.

El ancho de las costas queda definido por patrones climatológicos dominados por el oleaje donde las corrientes longitudinales promueven la erosión en las zonas proximales de las costas.

Los resultados demuestran que las costas responden episódicamente a las ocurrencias de grandes tormentas que producen grandes olas coincidentes con altos niveles 
de agua, y que consecuentemente tienen efectos directos en las infraestructuras, dunas, y humedales costeros.

La evolución morfológica costera es compleja porque los procesos hidromorfodinámicos varían en múltiples escalas espaciales, desde mecanismos de retroalimentación a microescala en el nivel de tamaño de grano del sedimento hasta períodos a escalas de tiempo geológicas. En el área de estudio, las condiciones del clima del oleaje son determinantes, pero también lo son las condiciones topográficas, orientación y exposición de las playas. Los procesos de oleaje pueden describir cambios en intervalos muy cortos de tiempo. Las acciones antropogénicas pueden estar acelerando procesos erosivos sin precedentes.

Las condiciones climáticas y cambios que está padeciendo por megasequías la región central de Chile, son poco conocidos y su impacto en el equilibrio de los sedimentos costeros, aún no ha podido ser estudiada.

\section{Agradecimientos}

Este trabajo ha sido financiado por la Agencia Nacional de Investigación y Desarrollo (ANID) a través del FONDEF IDeA I+D 2019 - Desafío País Adaptación al Cambio Climático y Desastres Naturales. Código Proyecto ID19I10361. Agradecimiento a los practicantes de Escuela de Geología de la Universidad Mayor por su colaboración en la toma de datos en campo y procesamiento de datos sedimentarios. Álvaro Millamán, Maximiliano Parrao, Catalina Cerda, Nicolás Rodríguez, a las asistentes, Natalia Medina, Roxana Mansilla. Al IHC Cantabria que nos ha cedido datos de oleaje y marea de la zona.

\section{References}

AGREDANO, R., CIENFUEGOS, R., CATALÁN, P., MIGNOT, E., BONNETON, P., BONNETON, N., and MARTÍNEZ, C.. 2019. Morphological changes in a cuspate sandy beach under persistent high-energy swells: Reñaca Beach (Chile). Marine Geology, 417(June 2018), 105988. DOI: 10.1016/j.margeo.2019.105988

AGUIRRE, C., BECERRA, D., GODOY, M., and SILVA, D., 2020. Interannual variability of ocean surface waves in the Southeast Pacific. In EGU General Assembly Conference Abstracts (p. 11567).

ALMONACID-CABALLER, J., SÁNCHEZ-GARCÍA, E., PARDO-PASCUAL, J. E., BALAGUER-BESER, A.A., and PALOMAR-VÁZQUEZ, J., 2016. Evaluation of annual mean shoreline position deduced from Landsat imagery as a mid-term coastal evolution indicator. Marine Geology, 372, pp. 79-88. DOI: 10.1016/j.margeo.2015.12.015

BEYÁ, J., ÁlVAREZ, M., GALLARDO, A., HIDALGO, H., AGUIRRE, C., VALDIVIA, J., and WINCKLER, P., 2016. Atlas de oleaje de Chile. Escuela de Ingeniería Civil Oceánica, Universidad de Valparaíso, Valparaíso.

CAMPOS-CABA, R. V., 2016. Análisis de marejadas históricas y recientes en las costas de Chile. Universidad de Valparaíso. Valparaíso.

CABEZAS-RABADÁN, C., PARDO-PASCUAL, J. E., ALMONACID-CABALLER, J., and RODILLA, M., 2019. Detecting problematic beach widths for the recreational function along the Gulf of Valencia (Spain) from Landsat 8 subpixel shorelines. Applied Geography, 110(July), 102047. DOI: 10.1016/j.apgeog.2019.102047

CABEZAS-RABADÁN, C., ALMONACID-CABALLER, J., PARDO-PASCUAL, J. E., and SORIANO-GONZÁLEZ, J., 2017. Variabilidad de la línea de costa a partir de imágenes de satélite y su relación con la textura del sedimento. In Primer Congreso en Ingeniería Geomática. Libro de actas (pp. 153-161). Editorial Universitat Politècnica de València.

CABEZAS-RABADÁN, C. and PARDO-PASCUAL, J. E., 2017. Monitorizando la anchura de las playas mediante imágenes Landsat 8 en costas micromareales mediterráneas. Geotemas (Madrid), 17, pp. 159-162.

CHAWLA, A., SPINDLER, D. M., and TOLMAN, H. L., 2013. Validation of a thirty year wave hindcast using the Climate Forecast System Reanalysis winds. Ocean Modelling, 70, pp. 189-206. DOI: 10.1016/j.ocemod.2012.07.005

FEYISA, G. L., MEILBY, H., FENSHOLT, R., and PROUD, S. R., 2014. Automated Water Extraction Index: A new technique for surface water mapping using Landsat imagery. Remote Sensing of Environment, 140, pp. 23-35. DOI: 10.1016/j.rse.2013.08.029

GARREAUD, R. D., BOISIER, J. P., RONDANELLI, R., MONTECINOS, A., SEPÚLVEDA, H. H., and VELOSO-AGUILA, D., 2020. The Central Chile Mega Drought (2010-2018): A climate dynamics perspective. International Journal of Climatology, 40(1), pp. 421-439. DOI: 10.1002/joc.6219

GENS, R., 2010. Remote sensing of coastlines: Detection, extraction and monitoring. International Journal of Remote Sensing, 31(7), pp. 1819-1836. DOI: 10.1080/01431160902926673

HEMER, M. A., CHURCH, J. A., and HUNTER, J. R., 2010. Variability and trends in the directional wave climate of the Southern Hemisphere. International Journal of Climatology, 30(4), pp. 475-491. DOI: 10.1002/joc.1900

HERSBACH, H., BELL, B., BERRISFORD, P., HIRAHARA, S., HORÁNYI, A., MUÑOZ-SABATER, J., and SCHEPERS, D., 2020. The ERA5 global reanalysis. Quarterly Journal of the Royal Meteorological Society, 146(730), pp. 19992049.

IGUALT JARA, F. E., BREUER, W. A., CONTRERAS-LÓPEZ, M., and MARTíNEZ, C., 2019. Efectos del cambio climático en la zona urbana turística y costera de Viña del Mar: levantamiento de daños para una inundación por marejadas y percepción de seguridad. Revista 180, 44. DOI: 10.32995/rev180.num-44.(2019).art-626 
KIM, H., LEE, S. B., and MIN, K. S., 2017. Shoreline change analysis using airborne LiDAR bathymetry for coastal monitoring. Journal of Coastal Research, 33(Special Issue 79), pp. 269-273. DOI: 10.2112/SI79-055.1

LIU, Y., WANG, X., LING, F., XU, S., and WANG, C., 2017. Analysis of coastline extraction from Landsat-8 OLI imagery. Water (Switzerland), 9(11), pp. 1-26. DOI: 10.3390/w9110816

MARTíNEZ, C., 2007. Shoreline changes in Concón and Algarrobo bays, central Chile, using an adjustment model. Investigaciones Marinas, 35(2), pp. 99-112. DOI: 10.4067/s0717-71782007000200010

MARTíNEZ, C., 2011. Análisis comparativo de cambios históricos de la línea litoral en bahías chilenas: orientaciones al manejo costero. Revista Geográfica de América Central, 2(47E).

MARTÍNEZ, C., ARENAS, F., BERGAMINI, K., and URREA, J., 2019. Hacia una ley de costas en Chile : criterios y desafíos en un contexto de cambio climático. (CIGIDEN, Ed.), Serie policy papers cigiden (1st ed.). Santiago, Chile.

MARTíNEZ, C., CONTRERAS-LÓPEZ, M., WINCKLER, P., HIDALGO, H., GODOY, E., and AGREDANO, R., 2018. Coastal erosion in central Chile: A new hazard? Ocean and Coastal Management, 156, pp. 141-155. DOI: 10.1016/j.ocecoaman.2017.07.011

MARTÍNEZ, C., ROJAS, C., ROJAS, O., QUEZADA, J., LÓPEZ, P., and RUÍZ, V., 2016. Crecimiento urbano sobre geoformas costeras de la llanura de San Pedro, area metropolitana de Concepcion. En Las Costas Del Neoliberalismo, pp. 287-312.

MAS, J. F., 2004. Mapping land use/cover in a tropical coastal area using satellite sensor data, GIS and artificial neural networks. Estuarine, Coastal and Shelf Science, 59(2), pp. 219-230. DOI: 10.1016/j.ecss.2003.08.011

MASOTTI, I., APARICIO-RIZZO, P., YEVENES, M. A., GARREAUD, R., BELMAR, L., and FARÍAS, L., 2018. The influence of river discharge on nutrient export and phytoplankton biomass off the Central Chile Coast $\left(33^{\circ}-37^{\circ} \mathrm{S}\right)$ : Seasonal cycle and interannual variability. Frontiers in Marine Science, 5(NOV), pp. 1-12. DOI: 10.3389/fmars.2018.00423

MASSELINK, G., and PATTIARATCHI, C. B., 2001. Seasonal changes in beach morphology along the sheltered coastline of Perth, Western Australia. Marine Geology, 172(3-4), pp. 243-263. DOI: 10.1016/S0025-3227(00)00128-6

MINISTERIO DEL MEDIO AMBIENTE, 2019. Amenazas en "Determinación del riesgo de los impactos del Cambio Climático en las costas de Chile” (Vol. 1). Santiago, Chile.

NICHOLLS, R. J., 1995. Coastal megacities and climate change. GeoJournal, 37(3), pp. 369-379. DOI: 10.1007/BF00814018

PALOMAR-VÁZQUEZ, J., ALMONACID-CABALLER, J., PARDO-PASCUAL, J. E., and SANCHEZ-GARCÍA, E., 2018. SHOREX: a new tool for automatic and massive extraction of shorelines from Landsat and Sentinel 2 imagery. In 7 th International Conference on the Application of Physical Modelling in Coastal and Port Engineering and Science (Coastlab). Santander.

PARDO-PASCUAL, J. E., ALMONACID-CABALLER, J., RUIZ, L. A., and PALOMAR-VÁZQUEZ, J., 2012. Remote Sensing of Environment Automatic extraction of shorelines from Landsat TM and ETM + multi-temporal images with subpixel precision. Remote Sensing of Environment, 123, pp. 1-11. DOI: 10.1016/j.rse.2012.02.024

PARDO-PASCUAL, J. E., SÁNCHEZ-GARCÍA, E., ALMONACID-CABALLER, J., PALOMAR-VÁZQUEZ, J. M., DE LOS SANTOS, E. P., FERNÁNDEZ-SARRÍA, A., and BALAGUER-BESER, Á., 2018. Assessing the accuracy of automatically extracted shorelines on microtidal beaches from landsat 7, landsat 8 and sentinel-2 imagery. Remote Sensing, 10(2), pp. 1-20. DOI: 10.3390/rs10020326

PÉREZ, J., MENENDEZ, M., and LOSADA, I. J., 2017. GOW2: A global wave hindcast for coastal applications. Coastal Engineering, 124(January), pp. 1-11. DOI: 10.1016/j.coastaleng.2017.03.005

RANGEL-BUITRAGO, N., CONTRERAS-LÓPEZ, M., MARTÍNEZ, C., and WILLIAMS, A., 2018. Can coastal scenery be managed? The Valparaíso region, Chile as a case study. Ocean and Coastal Management, 163(April), pp. 383-400. DOI: 10.1016/j.ocecoaman.2018.07.016

SÁNCHEZ-GARCÍA, E., PALOMAR-VÁZQUEZ, J. M., PARDO-PASCUAL, J. E., ALMONACID-CABALLER, J., CABEZAS-RABADÁN, C., and GÓMEZ-PUJOL, L., 2020. An efficient protocol for accurate and massive shoreline definition from mid-resolution satellite imagery. Coastal Engineering, 160, $103732 . \quad$ DOI: 10.1016/j.coastaleng.2020.103732

SÁNCHEZ-GARCÍA, ELENA, BALAGUER-BESER, Á., ALMONACID-CABALLER, J., and PARDO-PASCUAL, J. E., 2019. A new adaptive image interpolation method to define the shoreline at sub-pixel level. Remote Sensing, 11(16). DOI: $10.3390 /$ rs 11161880

SCOTT, J. W., MOORE, L. R., and HARRIS, W. M., 2003. Using the Landsat 7 Enhanced Thematic Mapper Tasseled Cap Transformation to Extract Shoreline. US Geological Survey Open-File Report, (March).

SEKOVSKI, I., STECCHI, F., MANCINI, F., and DEL RIO, L., 2014. Image classification methods applied to shoreline extraction on very high-resolution multispectral imagery. International Journal of Remote Sensing, 35(10), pp. 35563578. DOI: 10.1080/01431161.2014.907939 
STOPA, J. E., and CHEUNG, K.FAI., 2014. Periodicity and patterns of ocean wind and wave climate. Journal of Geophysical Research: Oceans, 119, pp. 3868-3882. DOI: 10.1002/2013JC009729.Received

SUNDER, S., RAMSANKARAN, R., and RAMAKRISHNAN, B., 2017. Inter-comparison of remote sensing sensing-based shoreline mapping techniques at different coastal stretches of India. Environmental Monitoring and Assessment, 189(6), 290.

VOS, K., HARLEY, M. D., SPLINTER, K. D., SIMMONS, J. A., and TURNER, I. L., 2019. Sub-annual to multi-decadal shoreline variability from publicly available satellite imagery. Coastal Engineering, 150(April), pp. 160-174. DOI: 10.1016/j.coastaleng.2019.04.004

WIJNBERG, K. M., 2002. Environmental controls on decadal morphologic behaviour of the Holland coast. Marine Geology, 189(3-4), pp. 227-247. DOI: 10.1016/S0025-3227(02)00480-2

WINCKLER, P., CONTRERAS-LÓPEZ, M., CAMPOS-CABA, R., BEYÁ, J. F., and MOLINA, M., 2017. El temporal del 8 de agosto de 2015 en las regiones de Valparaíso y Coquimbo, Chile Central. Latin American Journal of Aquatic Research, 45(4), pp. 622-648.

ZANUTTA, A., LAMBERTINI, A., and VITTUARI, L., 2020. UAV photogrammetry and ground surveys as a mapping tool for quickly monitoring shoreline and beach changes. Journal of Marine Science and Engineering, 8(1). DOI: 10.3390/JMSE8010052 